\title{
Comparison between Functional Outcome of Arthroscopic Debridement Versus Arthroscopic Repair of Incomplete Thickness Tear of Shoulder Rotator Cuff H.El-Begawim ${ }^{1}$, A.S.El-Hammady ${ }^{1}$, S.A.El Tregy ${ }^{1}$ and M.O.Rizk ${ }^{1}$ Orthopedic Surgery Dept., Faculty of Medicine, Benha Univ., Benha, Egypt E-Mail: Rizk55@gmail.com
}

\begin{abstract}
The main line of treatment of halfway rotator sleeve tears is normally non-employable. This incorporates action alteration, NSAIDs and torment meds, physiotherapy, and steroid infusion. Not at all like full thickness rotator sleeve tears, the danger of greasy invasion, strong decay, and critical tear augmentation are generally insignificant. Be that as it may, the achievement of non-employable treatment of incomplete rotator sleeve tear has once in a while been accounted for. Careful treatment of Partial rotator sleeve tear can be utilized after disappointment of preservationist the board. twenty patient was partitioned into two treatment bunches for examination. Ten patients were dealt with arthroscopic debridement and sub-acromial decompression just while ten patients were overseen by arthroscopic debridement and sub-acromial decompression and in-situ fix of the rotator sleeve by anchors.All patients began physiotherapy program with a particular comparative convention. Patients were assessed utilizing UCLA scoring at 3 and a half year. At a quarter of a year development, acceptable outcomes dependent on the patient's fulfillment was $70 \%$ of the all out gathering and following a half year follow-up a fulfillment accomplished of $100 \%$ of the patients. In view of the UCLA scoring, arthroscopic debridement had normal improvement from mean UCLA scoring of 12.8 pre-operatively to 31.6 following a half year. The result for the entire gathering of patients with PTRCT after disappointment of preservationist treatment and high-grade torment meddling with day by day life movement show high critical incentive following a half year of follow-up utilizing UCLA scoring framework for appraisal and assessment.
\end{abstract}

\section{Introduction}

The rotator sleeve is a complex of muscles that emerge from the scapula and whose ligaments mix in with the subjacent container as they append to the tuberosities of the humerus [1]. Rotator sleeve tears are a typical orthopedic issue, and regularly these tears are alleged fractional tears of the rotator sleeve. An incomplete tear of the rotator sleeve is a zone of harm to the rotator sleeve ligaments, where the tear doesn't go right through the ligament [2].

In every clinical report, the occurrence of rotator sleeve fragmented imperfections is moderately low before the age of 40 years, starts to ascend in the 50-to 60-year mature age gathering and keeps on expanding in the 70 years and more established age group [3].

In a fractional rotator sleeve injury, the ligament is harmed, however not right through. The top piece of the ligament is in some cases harmed, the base piece of the ligament is harmed different occasions, and at times, the internal piece of the ligament can be harmed. Incomplete tears can be only a stage away from being a finished tear [3]. Surgical treatment of Partial rotator sleeve tear can be utilized after disappointment of preservationist management [4].

The point of the proposition was to think about the outcomes and practical result between arthroscopic debridement and arthroscopic fix of fractional thickness tear of rotator sleeve.
This is a Prospective randomized control preliminary on twenty patients who had deficient thickness shoulder rotator sleeve tear; they were alluded to the principle place emergency clinic of Egyptian Railway in Cairo. The investigation companion was partitioned into two treatment bunches for examination. Ten patients were made do with arthroscopic debridement and sub-acromial decompression just while ten patients were overseen by arthroscopic debridement and subacromial decompression and in-situ fix of the rotator sleeve by grapples.

\subsection{Inclusion criteria}

- Patients with aged grouped between 2060 years.

- Patients with traumatic or non-traumatic (degenerative) incomplete rotator cuff tear.

All patients were fit for arthroscopic surgery and fit for general anesthesia, and all have been informed about surgical details and other non-surgical alternatives.

\subsection{Exclusion criteria}

- Patients younger than 20 years or Older than 60 years.

- Patients with recurrent rotator cuff tear.

- Complete or massive rotator cuff tear.

- Patients who were unfit for general anesthesia.

- Patients refused to join the study after explaining risks and benefits.

\section{Patient and method}


This is a Prospective randomized control preliminary on twenty patients who had deficient thickness shoulder rotator sleeve tear; they were alluded to the principle place emergency clinic of Egyptian Railway in Cairo. The investigation companion was partitioned into two treatment bunches for examination. Ten patients were made do with arthroscopic debridement and sub-acromial decompression just while ten patients were overseen by arthroscopic debridement and subacromial decompression and in-situ fix of the rotator sleeve by grapples.

\section{UCLA scoring (University of California Los Angeles)}

The score is a combination of physical exam findings (active forward elevation and strength) and subjective patient-reported measures (pain, satisfaction, and function). Pain and function are preferentially weighted 20 out of 35 possible points. A higher score indicates better function.

The collected data was revised, coded, tabulated and introduced to a PC using Statistical package for Social Science (SPSS 25). Data was presented and suitable analysis was done according to the type of data obtained for each parameter.

\section{Results}

In this study, group (1) had a mean age value of 44.4. Group (2) age mean value was 51.8. Each group had 1 female patient out of the 5 patients in each group and 1 patient being affected at the non-dominant side for each group.

Regarding pain, Group (1) pre-operatively had 4 patients presented with pain during light activity (40\%) and 6 patients with pain that is always present but bearable with strong analgesics (60\%). Group (2) pre-operative had 2 patients with pain during light activities $(20 \%)$ and 8 patients with pain that is always present but bearable with strong analgesics $(80 \%)$.

At three months post-operative, group (1) had 4 patients with pain during light activity $(40 \%)$ and 6 patients with pain during heavy activities (60\%). On the other hand, group (2) had 2 patients $(20 \%)$ with pain that is always present but bearable and 2 patient (20\%) with pain during light activities. There were 2 patients (20\%) with pain during heavy activities and $4(40 \%)$ patients with occasional pain.

The p-value between the two groups was 1.00 at three months post-operative pain with non-significant value. Six months postoperative, group (1) had 2 patients $(20 \%)$ with pain during light activities and 6(60\%) patient with occasional pain while 2 pain had no pain (20\%). Group (2) had $2(20 \%)$ patient with pain during light activities and 8 (80\%) patients with occasional pain only. The pvalue between the two groups was 1.00 at six months post-operative pain with nonsignificant value.

Active forward flexion, pre-operatively Group (1) had 2 patients (20\%) with active forward flexion between 450-900, 6 patients (60\%) between $900-1200$ and 2 patients (20\%) 1200-1500. Group (2) had 2 patients (20\%) of active forward flexion $450-900$ and 8 patients between 900-1200.

Three months post-operative, all patient in group (1) had active forward flexion $>1500$ $(100 \%)$ while group (2) had 6 patients $(60 \%)$ with active forward flexion 1200-1500 and 4 patients $(40 \%)>1500$. The p-value between the two groups was 0.167 at three months postoperative active forward flexion which shows a non-significant value.

Six months post-operative, all patient in both groups had active forward flexion $>1500$.

In this study, regarding satisfaction, all patients pre-operatively in both groups were not satisfied. Three months post-operative there was 2 patients dissatisfied in group (1) while there were 4 patients not satisfied in groups (2). The p-value is 1.00 with nonsignificant value. Six months post-operative all patients were satisfied.

Function pre-operative, group (1) had 2 patients (20\%) with light activity and 8 patients $(80 \%)$ with only light house work. Group (2) all patients with only light house work functionality.

Three months post-operative, group (1) had 4 patients $(40 \%)$ were able to do most house work and 6 patients $(60 \%)$ with only slight restrictions. Group (2) had $2(20 \%)$ patient were able to do only light house work and 8 (80) patients with most of the house work. The p-value of functioning at three months postoperative was 0.167 with no significant value.

At six months post-operative, group (1) had $4(40 \%)$ patients with only slight restrictions regarding their functioning and $6(60 \%)$ patients with completely normal activity. All patients in group (2) returned to their normal activities within 6 months post-operative. The p-value of functioning at six months postoperative was 0.444 with no significant value.

For Strength of forward flexion, preoperatively, group (1) had 8 patients with grade III $(80 \%)$ and 2 patients $(20 \%)$ with grade IV. Group (2) had 6 patients (60\%) with grade III and 4 patients (40\%) with grade VI.

Three months post-operative, in group (1) there was 2 patients (20\%) with strength of 
active forward flexion grade III and 4 patients with grade IV and V respectively with $40 \%$ for each group. Group (2) had 2 patients (20\%) with grade III, 6 patients $(60 \%)$ with grade IV and 2 patients $(20 \%)$ with grade $\mathrm{V}$. The pvalue of strength of forward flexion at three months post-operative was 1.00 with no significant value.

Six months post-operative, all patients had strength of active forward flexion grade $\mathrm{V}$ in the two groups.

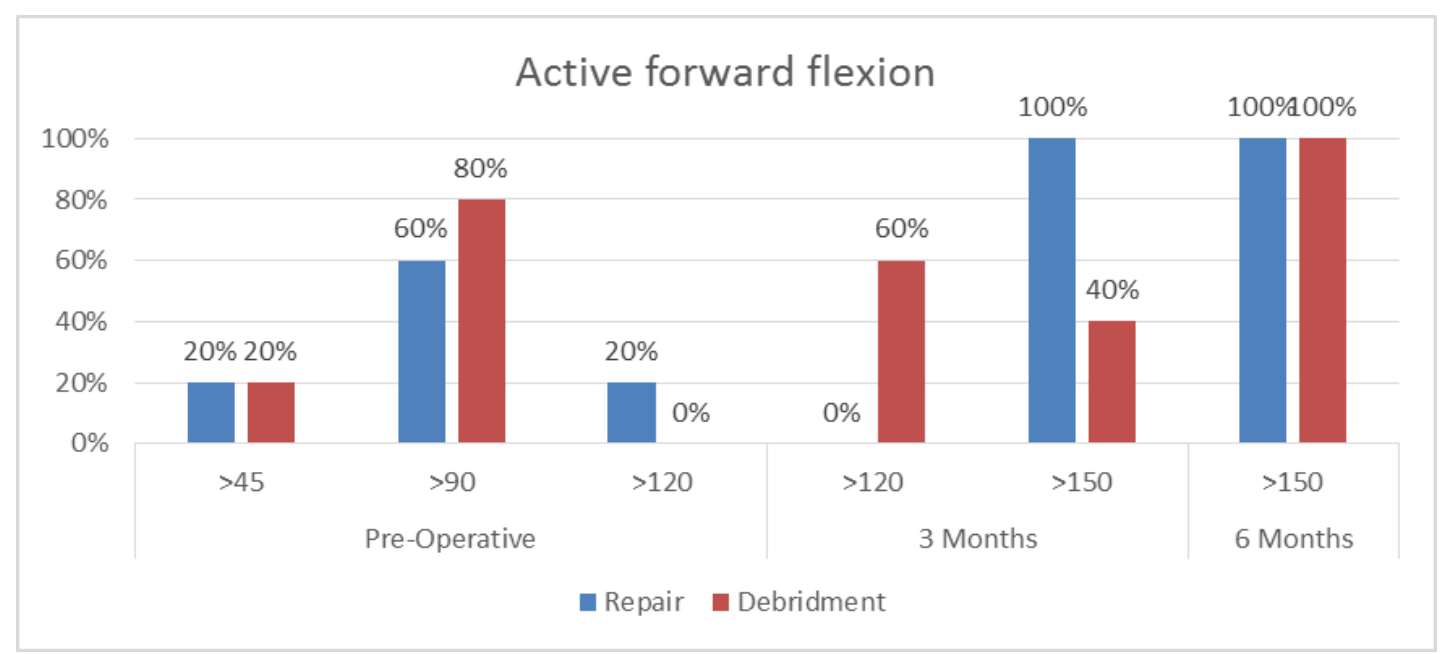

Fig (1) Active forward flexion comparative illustrated graph between the two groups.

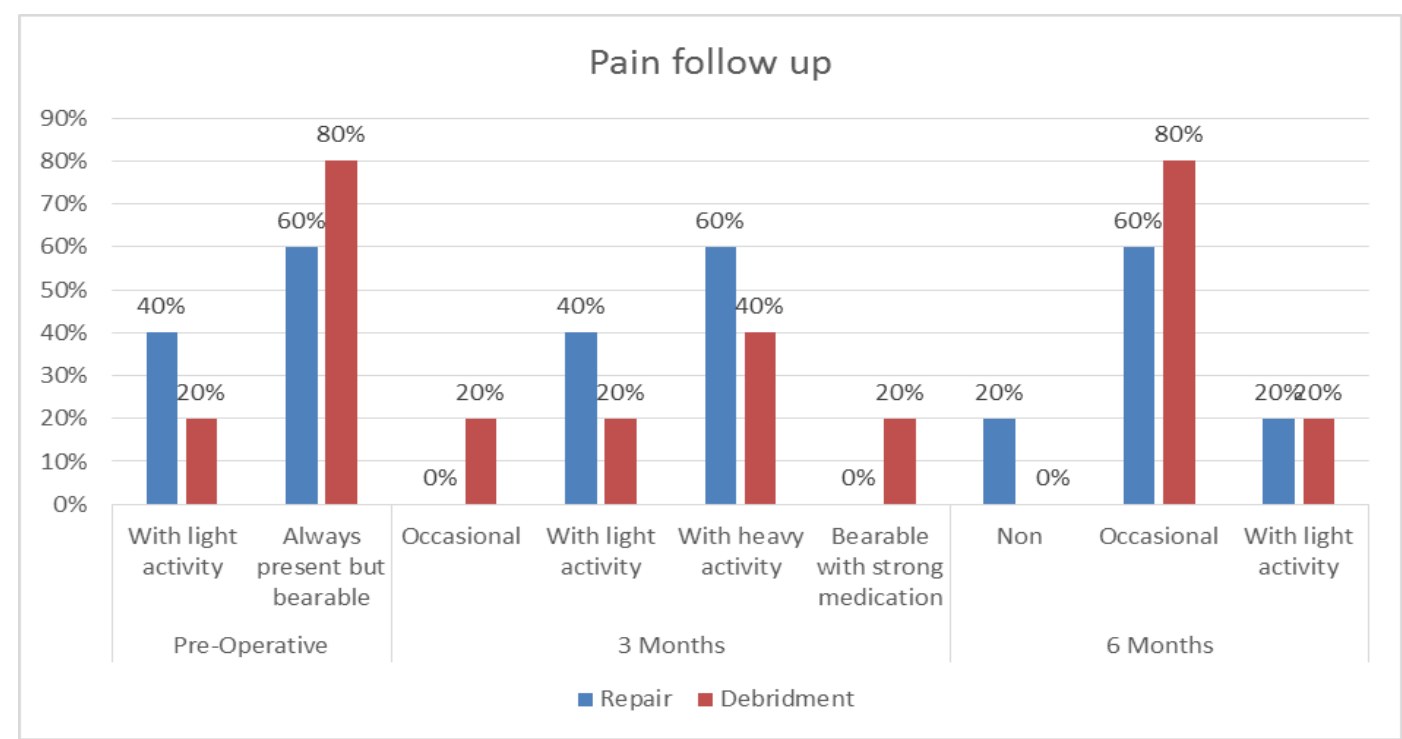

Fig (2) Pain measurement comparative illustrated graph between the two groups.

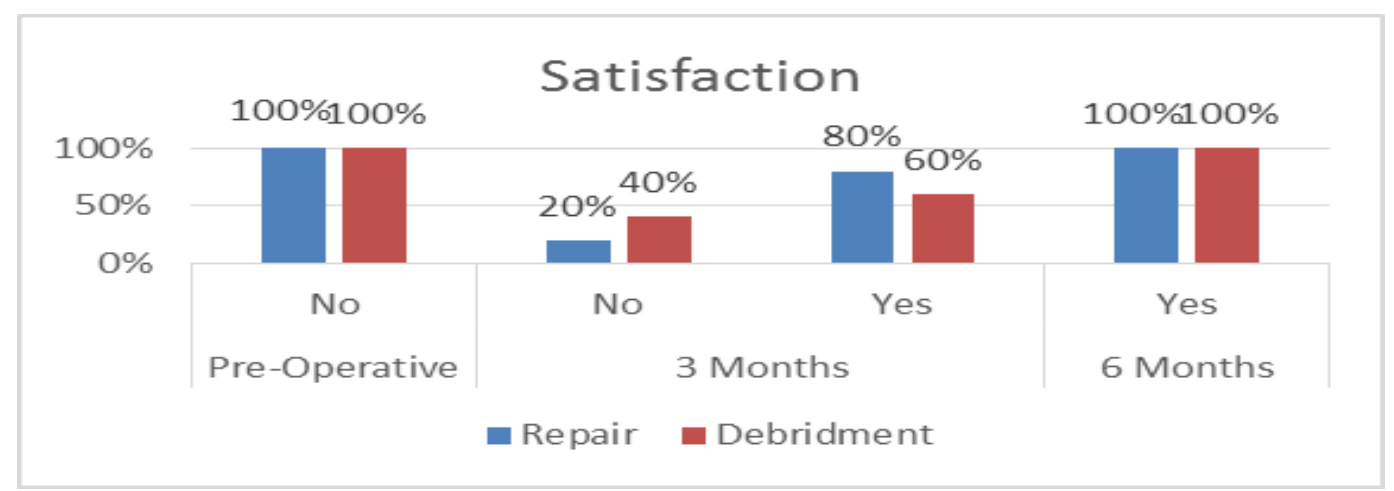

Fig (3) Satisfaction comparative illustrated graph between the two groups. 


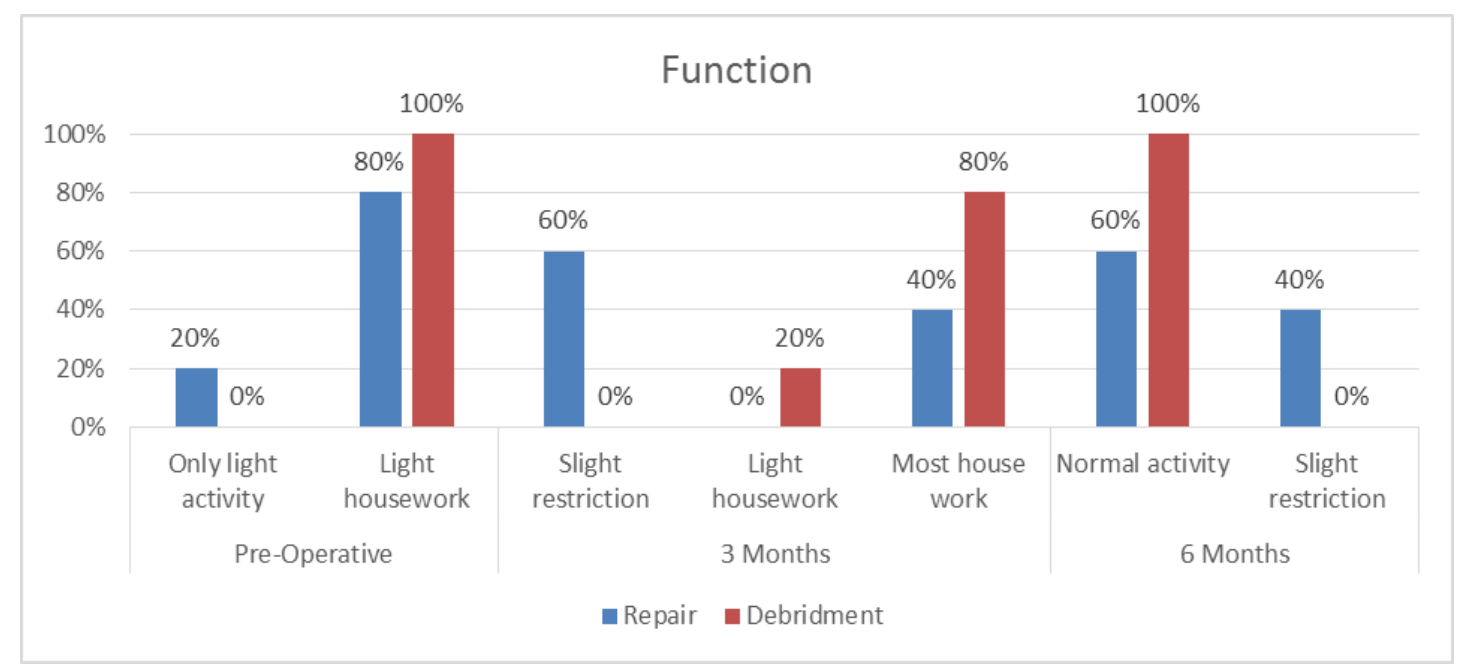

Fig (4) Function comparative illustrated graph between the two groups.

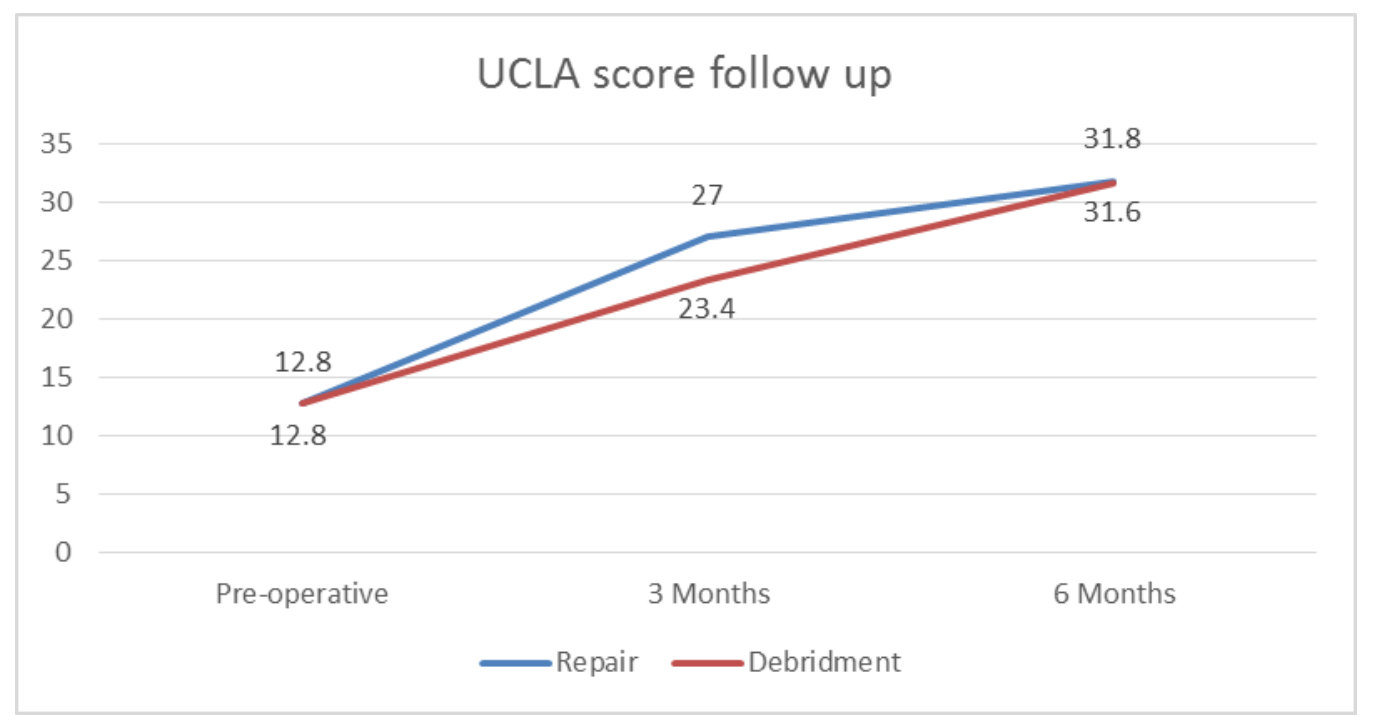

Fig (5) UCLA score follow up between the two groups.

\section{Discussion}

In the current examination, twenty patients were assessed and determined to have incomplete thickness tear rotator sleeve of the shoulder and were dealt with arthroscopically either with debridement alone, or with arthroscopic fix by grapple.

At a quarter of a year development, acceptable outcomes dependent on the patient's fulfillment was $70 \%$ of the all out gathering. In any case, following a half year follow-up a fulfillment accomplished of $100 \%$ of the patients.

In light of the UCLA scoring, arthroscopic debridement had normal improvement from mean UCLA scoring of 12.8 pre-operatively to 31.6 following a half year.
In spite of the fact that Budoff et al (5) assessed a bigger number of patients than the current examination, comparable outcomes dependent on the finding and treatment of 79 shoulders with halfway thickness sleeve tears rewarded with arthroscopic debridement alone after a subsequent period extending from 25 to 93 months.

Of note, a comparable outcome to our investigation, utilizing the University of California at Los Angeles (UCLA) Shoulder Rating Scale, the consequences of debridement alone did not regard superb in $89 \%$ of those with under 5 years of development. In those with follow-up more prominent than 5 years, the outcomes diminished to $81 \%$ [6].

Andrews et al [7] revealed $85 \%$ great and amazing outcomes with arthroscopic 
debridement of fractional thickness tears in 34 youthful competitors with a mean follow-up of 13 months.

In the current investigation, every one of our patients performed acromioplasty. Snyder et al [6] reflectively checked on 31 patients with fractional thickness tears rewarded in this design and revealed $84 \%$ great to superb outcomes. In any case, 13 of the 31 patients didn't experience subacromial decompression. Strikingly, no noteworthy distinction was found in the result, whether or not decompression was performed.

Esch et al [8] assessed the consequences of arthroscopic subacromial decompression as per the level of the tear. Seventy-six percent of the patients with a fractional thickness tear showed good scores on the UCLA Shoulder Rating Scale, with an improvement in torment, work, dynamic forward flexion, and quality. Understanding fulfillment was exhibited in $82 \%$ of these patients.

In spite of the fact that Snyder's outcomes indicated no critical distinction whether decompression is done or not, Cordasco et al [9] assessed the clinical result of arthroscopic acromioplasty and debridement in 162 patients with ordinary or incomplete thickness tears of the rotator sleeve. They noticed an expanded disappointment rate in patients with grade $2 \mathrm{~B}$ (bursal-sided tears influencing under half of ligament thickness). Therefore, they suggested mix with acromioplasty for these specific tears.

So also, Payne et al [10] assessed 43 competitors more youthful than 40 years with incomplete thickness tears rewarded with arthroscopic debridement and subacromial decompression. Those with intense, horrible wounds had a good result $86 \%$ of the time, with an arrival to preinjury sports in $64 \%$.

In our investigation, the mean of the UCLA scoring of arthroscopic fix bunch was 12.8 preoperatively and had huge improvement to 31.8 following a half year and All patients were fulfilled at a half year of development

Conway [11] had comparable outcomes which revealed a $89 \%$ come back to the equivalent or more elevated level of play in a gathering of 14 baseball players who experienced fix of Intratendinous rotator sleeve tears.

A comparative report Park et al [12] thought about the aftereffects of arthroscopic fix of patients who had fractional thickness rotator sleeve tears with those of patients who had full-thickness tears. After a mean followup of 34 months, the two gatherings showed comparable enhancements in agony, movement, and capacity by the American Shoulder and Elbow Society Score.
Assessment at last follow-up indicated that $93 \%$ of all patients had great or astounding outcomes, and $95 \%$ showed agreeable result with respect to torment decrease and utilitarian result.

An investigation of fix methods by Lo and Burkhart [13] presented the possibility of a contorted length-strain relationship of the supraspinatus ligament after transformation of a PTRCT to a full-thickness tear and proposed progressively great results of a trans-ligament in situ fix. Current proof in the writing, in any case, proposes that no distinctions can be found at present moment follow-up among auxiliary and practical results and difficulty paces of in situ fix versus fix after change of evaluation 3 PTRCTs to full-thickness tears.

Relative examination in our investigation between the patients performed arthroscopic debridement and the patients performed arthroscopic fix indicated no huge qualities dependent on UCLA scoring. Comparative outcomes were accomplished in the two gatherings in things of torment, fulfillment, scope of movement, muscle quality and day by day life exercises.

Weber [14] reflectively looked into 2 comparable gatherings of patients with incomplete thickness rotator sleeve tears who were watched for 2 to 7 years. One gathering was treated with arthroscopic debridement and acromioplasty with 14 great and no phenomenal outcomes. The subsequent gathering experienced arthroscopic fix and acromioplasty with 28 great and 3 fantastic outcomes. Because of this examination, Weber suggested fix of fractional thickness tears more noteworthy than half of the ligament thickness. Be that as it may, an exact technique to survey sleeve thickness, the impact of shifting individual ligament measurements, and forthcoming affirmation of this suggestion stay to be resolved.

Late work by Nicholson et al. [15] utilized $30 \%$ tear profundity as a limit for fix. Inactive patients may do well with decompression alone, even in the setting of a tear half of the profundity of the rotator sleeve, though dynamic patients will probably profit by an increasingly forceful methodology with an endeavored fix for a tear that is somewhere in the range of $30 \%$ and half of sleeve profundity.

A forceful endeavor at fix is additionally demonstrated in those patients with an intense, horrible tear and in those whose tear is on the bursal surface on account of the good blood flexibly of this area and the for the most part helpless result related with debridement of bursal-sided tears [15]. 


\section{Conclusion}

Until this point, the profundity of the tear as indicated by Ellman, the evaluation of tendinosis (which was to some degree identified with the tear size), and the area of the tear (bursal versus articular) have been recognized as significant tear attributes of PTRCTs. It stays hazy, be that as it may, if and how the foremost back augmentation (length) of the tear, the basic shoulder point and the ligament quality (greasy penetration and decay) influence utilitarian and auxiliary results [16].

\section{References}

[1] J .Rudez, M. Zanetti, Normal anatomy, variants and pitfalls on shoulder MRI. Eur J Radiol, Vol.68(1), PP. 25-35,2008.

[2] T.S. Cook, J.M. Stein, S . Simonson, Normal and variant anatomy of the shoulder on MRI. MagnReson Imaging Clin N Am, Vol.19, PP. 581-594,2011.

[3] S. Tempelhof, S. Rupp, R . Seil ,Agerelated prevalence of rotator cuff tears in asymptomatic shoulder. J Shoulder Elbow Surg, Vol. 8, PP.296-299,2009.

[4] H. Ellman , "Diagnosis and treatment of incomplete rotator cuff tears," Clinical Orthopedics and Related Research, Vol. 254, PP.64-74,1990.

[5] J. E. Budoff, D. Rodin, D. Ochiai , "Arthroscopic rotator cuff debridement without decompression for the treatment of tendinosis," Arthroscopy, Vol. 21(9), PP. 1081- 1089,2005.

[6] S.J. Snyder, A.F. Pachelli, W. Del Pizzo, Partial thickness rotator cuff tears: results of arthroscopic treatment. Arthroscopy, Vol. 7, PP.1-7,1991.

[7] D. Andrews, T.S. Broussard, W.G. Carson, Arthroscopy of the shoulder in the management of partial tears of the rotator cuff: a preliminary report.Arthroscopy,Vol1,pp.117-122, 2016.
[8] J.C. Esch, L.R. Ozerkis, J.A. Helgager, Arthroscopic subacromial decompression: results according to the degree of rotator cuff tear. Arthroscopy,Vol. 4, PP.241249,2001.

[9] F.A. Cordasco, M . Backer, E.V. Craig, The partial-thickness rotator cuff tear: is acromioplasty without repair sufficient? Am J Sports Med,Vol.30. PP.257260,2002.

[10]L.Z. Payne, D.W. Altchek, E.V. Craig, Arthroscopic treatment of partial rotator cuff tears in young athletes: a preliminary report. Am J Sports Med,Vol. 25, PP.299-305,2018.

[11] J.E. Conway, Arthroscopic repair of partial-thickness rotator cuff tears and SLAP lesions in professional baseball players. Orthop Clin North Am, Vol. 32, PP.443-456,2017.

[12] J.Y. Park, K.T. Chung, M.J. Yoo, A serial comparison of arthroscopic repairs for partial- and full-thickness rotator cuff tears. Arthroscopy, Vol.20, PP.705$711,2018$.

[13] S.S. Burkhart, Reconciling the paradox of rotator cuff repair versus debridement: a unified biomechanical rationale for the treatment of rotator cuff tears. Arthroscopy, Vol.10, PP.4-19,2007.

[14] S.C. Weber, Arthroscopic debridement and acromioplasty versus mini-open repair in the treatment of significant partialthickness rotator cuff tears Arthroscopy, Vol. 15, PP.126-131,2009.

[15]G.P. Nicholson, Arthroscopic acromioplasty: a comparison between workers' compensation and non-workers' compensation populations. J Bone Joint Surg Am, Vol. 85PP.682-689,2018.

[16] N.N. Shah, N.C. Bayliss , A. Malcolm Shape of the acromion: congenital or acquired-a macroscopic, radiographic, and microscopic study of acromion. J shoulder elbow surg, Vol.10, PP.309-316, 2001. 